\title{
EEG Coherence Analysis for Suppression of MEP Amplitude Variability in TMS
}

\author{
https://doi.org/10.3991/ijoe.v17i06.22553 \\ Keisuke Sasaki ${ }^{(\varpi)}$, Yuki Fujishige, Masato Odagaki \\ Maebashi Institute of Technology, Maebashi, Japan \\ k.s.gandhi@nifty.com
}

\begin{abstract}
Transcranial magnetic stimulation (TMS) is a non-invasive stimulation method for cortical neurons. When TMS is delivered to the primary motor cortex (M1), motor evoked potentials can be measured in electromyograms for the peripheral muscle. However, the motor-evoked potential (MEP) amplitudes measured by stimulations for M1 fluctuated from trial to trial. MEP fluctuations are caused by changes in cortical excitability. We hypothesized that MEP variability could be suppressed with application of TMS when cortical excitability was stable. Thus, we developed a TMS system to suppress MEP amplitude variabilities. We used electroencephalographic (EEG) online measurements with coherence analysis to obtain the similarity of cortical excitabilities. The system enables us to trigger TMS if the EEGs measured from the two channels have a high similarity in the frequency domain. In this study, we found that the suppression of MEP fluctuation was dependent on the state of cortical excitability obtained by EEG coherence analysis.
\end{abstract}

Keywords-Motor evoked potential, transcranial magnetic stimulation, coherence analysis, cortical excitability, frequency analysis

\section{Introduction}

Transcranial magnetic stimulation (TMS) over the primary motor cortex can elicit motor-evoked potentials (MEPs) in the electromyogram measured from a peripheral muscle [1]. The afferent signal from M1 to motor neurons through the corticospinal tract is delivered to the peripheral muscle after TMS stimulation [2]. MEP was measured at a latency of $20 \mathrm{~ms}$ following TMS. The MEP amplitude can be ordinarily observed with an intensity of approximately $1 \mathrm{mV}$. However, MEP fluctuation is often observed during the experiment even under constant stimulus conditions [3-8].

MEP elicited by the M1 stimulus is available to measure corticospinal excitability in a perioperative assessment for clinical application [9-12]. This fluctuation would affect the results of the evaluation of corticospinal excitability [13]. Thus, we needed to develop a measurement technique suitable for use in the clinical examination room to minimize MEP fluctuations.

Ogata et al. reported that the MEP amplitude depends on the frequency power spectrum density of the electroencephalogram (EEG) calculated by the fast Fourier 
transform (FFT). According to this report, the MEP amplitude was strengthened when the power of the $\alpha$ band spectrum was large. The power of the $\beta$ band does not affect the MEP amplitude [14]. In addition, several studies have shown that $\beta$ oscillations are inhibited when MEP amplitudes are large [15-18]. Because the intensity of MEP is related to the state of cortical activity [13], we assumed that the variability of the MEP could be suppressed after application of TMS over M1 when the cortical activity was stable. Our aim of this study is to develop the TMS navigation system for suppressing MEP fluctuation using online EEG measurements. We employed EEG coherence analysis to measure EEG stability using two channel electrodes [19]. EEG coherence analysis is generally used to measure the similarity of two or more signal waveforms in the frequency domain. Here, we conducted experiments to verify the validity of the proposed system. In the experiment, we measured the MEP variability and coherence values. We concluded that MEP variability could be suppressed with the stimulus condition based on the coherence value.

\section{Materials and Methods}

\subsection{TMS system for suppression of MEP variability}

We developed a TMS system to suppress MEP fluctuation. Figure 1 shows an overview of the proposed system. The system was composed of a TMS device (Magstim 200, Magstim Co. Ltd., UK), an EEG device (Polymate mini AP108, Miyuki Giken Co., Ltd, Japan), an EMG device (myoelectric sensor, Oisaka Electronics, Japan), and a personal computer with a data acquisition device (DAQ device: USB6210, National Instruments, USA). The EEG electrodes were placed at the locations of P3 and C4 with the international 10/20 system coordinates. Two EEGs were consecutively measured and analyzed. The TMS coil was placed over M1. MEP was measured with an EMG device with a sampling rate of $5 \mathrm{kHz}$, and then stored in the hard disk drive of the PC through the data acquisition board. The participants were asked to gaze at one point on the screen during the experiment. EEG was recorded at a sampling rate of $500 \mathrm{~Hz}$ and filtered using a fourth-order Butterworth bandpass filter with a cutoff frequency of $1 \mathrm{~Hz}$ to $30 \mathrm{~Hz}$. The software was written in MATLAB (Mathworks, Inc., USA), including the Data Acquisition Toolbox and Signal Processing Toolbox.

Figure 2 illustrates the experimental landscape. The subject comfortably sat on the chair and looked at one point on the monitor. The TMS coil was placed over the left M1, and MEP was recorded from the left index finger. Once the online EEG analysis starts, the system shows (a) the real-time EEG waveform and its power spectrum, as well as (b) the EEG coherence spectrum. After the TMS condition was satisfied, TMS was applied over M1 and the MEP waveforms were overlaid (c). The system can calculate the mean MEP amplitude and store all data of the EEG, the MEP waveform, and its stimulus condition into the hard disk drive. 


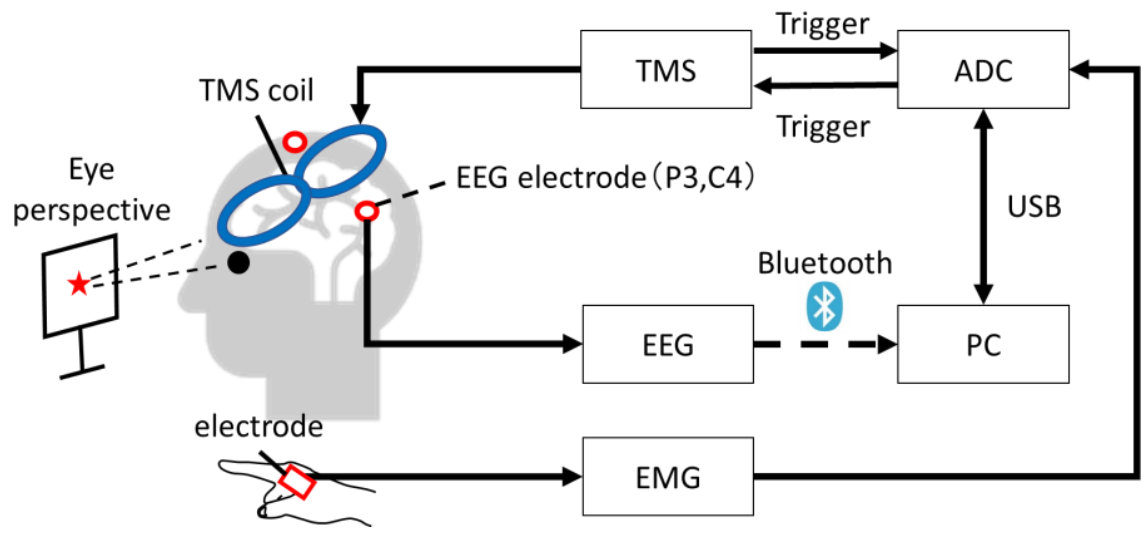

Fig. 1. TMS navigation system for suppressing MEP variability

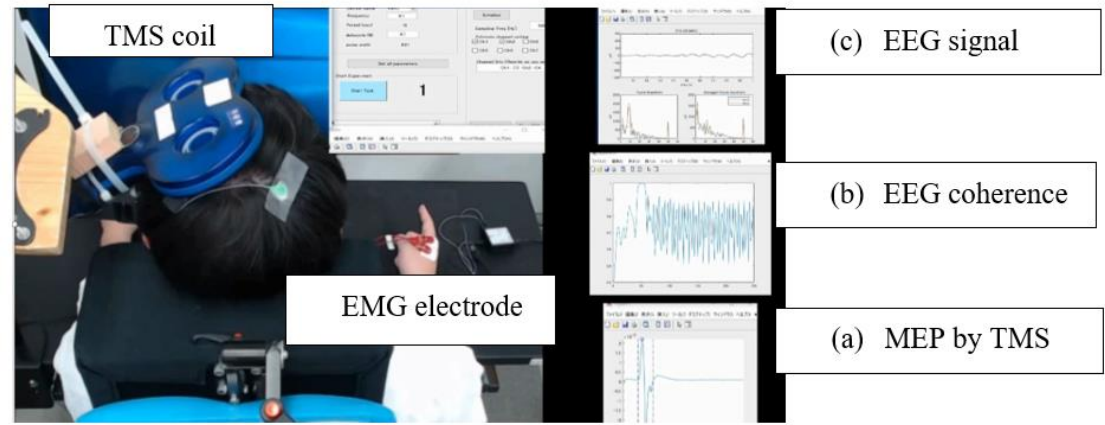

Fig. 2. Experimental landscape for online EEG analysis and TMS system. (a) EEG waveform and power spectrum, (b) EEG coherence value, (c) MEPs by TMS

\subsection{Coherence analysis of EEGs}

Coherence analysis was performed to measure the similarity between the two EEGs measured from P3 and C4. Coherence analysis can obtain the similarity between two waveforms as a frequency spectrum. We calculated the mean coherence values in the $\alpha$ and $\beta$ frequency bands as shown in Figure 3. The coherence function is defined as follows:

$$
\operatorname{coherence}(f)=\frac{\left|P_{P 3-C 4}(f)\right|^{2}}{P_{P 3}(f) P_{C 4}(f)}
$$

where coherence (f) is the power spectrum function of the similarity in the frequency domain. We calculated the coherence value using Eq. (1) with online EEG measurement and then calculated the similarity functions $\operatorname{Coh}(\alpha)$ and $\operatorname{Coh}(\beta)$ by the area of the coherence value between $8 \mathrm{~Hz}$ to $14 \mathrm{~Hz}$ and $14 \mathrm{~Hz}$ to $30 \mathrm{~Hz}$ using the following equations: 


$$
\begin{aligned}
& \operatorname{coh}(\alpha)=\operatorname{average}(\operatorname{coherence}(\alpha)) \\
& \operatorname{coh}(\beta)=\operatorname{average}(\operatorname{coherence}(\beta))
\end{aligned}
$$

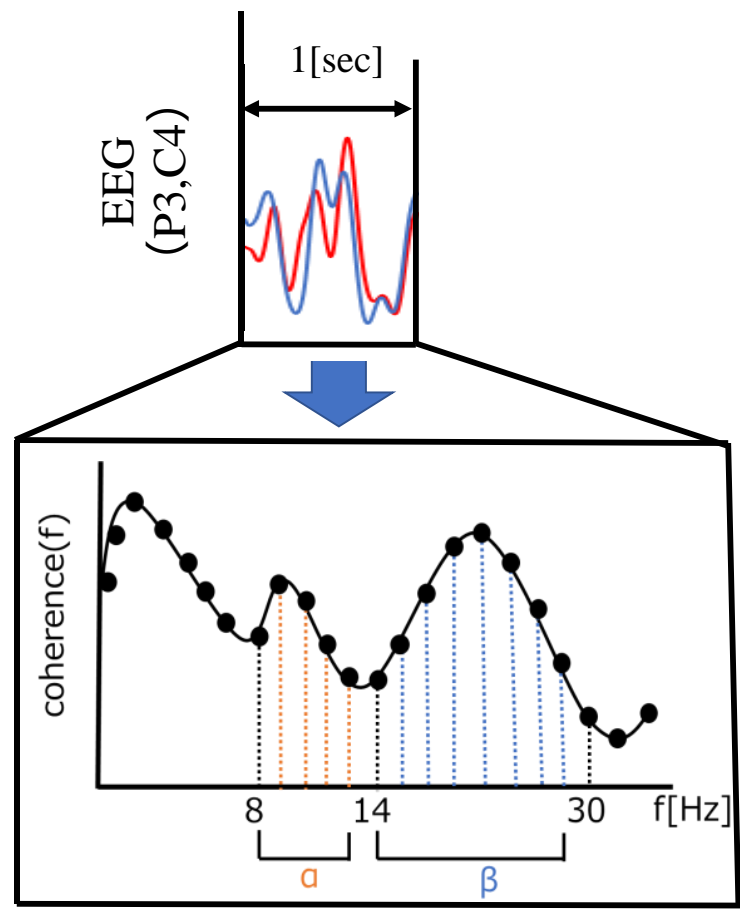

Fig. 3. Definitions of $\operatorname{coh}(\alpha)$ and $\operatorname{coh}(\beta)$. The frequency spectrum for coherence function was obtained from EEGs for $1 \mathrm{sec}$ and then the mean value was calculated in each frequency band.

\subsection{Experimental setup}

We confirmed the validity of the developed TMS system by measuring its coherence distribution. Seven healthy subjects participated in this experiment. Prior to the experiments, written informed consent was obtained from all subjects for publication. All procedures used in this study were approved by the Ethical Committee of the Maebashi Institute of Technology. Figure 4 shows the procedure for TMS experiments. TMS over the left M1 was applied 100 times at an interval of $15 \pm 3$ s. TMS intensity was set at $150 \%$ of the resting motor threshold. The resting motor threshold was defined as the intensity that was observed above $50 \mu \mathrm{V}$ with a probability of 50 $\%$. The subject gazed at one point on the screen and sustained the resting condition as much as possible. The MEPs were recorded from the first dorsal interosseous (FDI) muscle in every trial, and the amplitude of MEP peak-peak value was measured. EEG was measured from the electrodes of P3 and C4, and EEG coherence analysis was 
performed online. We then attempted to qualify the coherence value and show the distribution of MEP variability for each coherence condition.

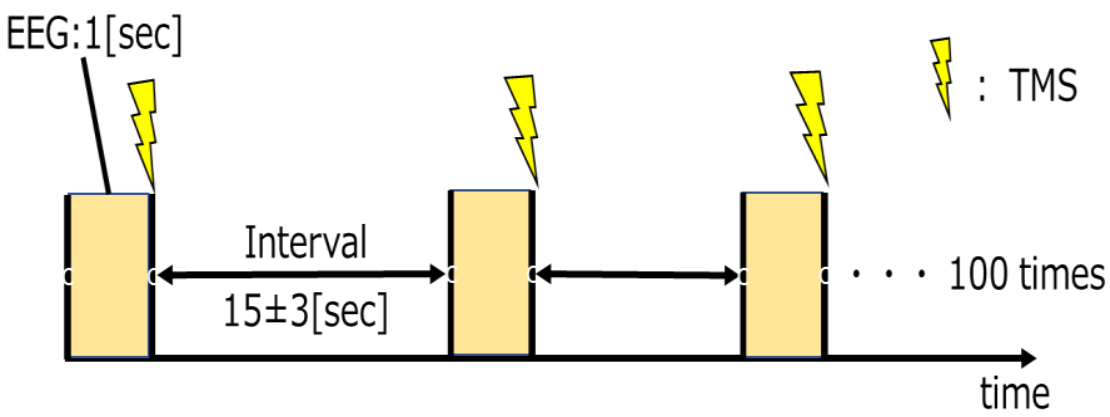

Fig. 4. Time sequence of TMS

\subsection{Analysis of MEP}

We attempted to detect the peak-peak voltage of the MEP and calculated the coefficient of variation (CV). The MEP normally appears approximately $20 \mathrm{~ms}$ after the TMS. CV was calculated using the following equation:

$$
C V=\frac{\sigma}{V}
$$

where $\sigma$ is the standard deviation of the MEP amplitude and V is the mean MEP value. CV expresses the variability of the measured values, and the data can be compared without considering the number of datapoints.

\section{$3 \quad$ Results and Discussions}

\subsection{Distribution of MEP coefficient of variation}

We created two color maps: the first for the number of MEP data and the second for the distribution of the coefficient of variation (CV) of MEP from each subject's data. Figures 5 and 6 show examples of the measurement results obtained from Subject 1 . Figure 5 shows a color map of the number of MEP data. The four-color maps were arranged such that the number of MEP data increased around the center of the figure. We categorized the MEP data with threshold values and showed a color map of the number of MEP data.

In the experiment, we set the four thresholds of the stimulus condition as follows: (1) $(\operatorname{Coh}(\alpha) \geq \alpha$ th, $\operatorname{Coh}(\beta) \leq \beta$ th $),(2)(\operatorname{Coh}(\alpha) \geq \alpha$ th, $\operatorname{Coh}(\beta) \geq \beta$ th $),(3)(\operatorname{Coh}(\alpha) \leq \alpha$ th, $\operatorname{Coh}(\beta) \leq \beta$ th $)$, and $(4)(\operatorname{Coh}(\alpha) \leq \alpha$ th, $\operatorname{Coh}(\beta) \geq \beta$ th $)$. Because the coherence values of $\operatorname{Coh}(\alpha)$ and $\operatorname{Coh}(\beta)$ should be in the range of 0 to 1 , the weakest conditions in each figure were determined as follows: 
1. $\operatorname{Coh}(\alpha) \geq 0, \operatorname{Coh}(\beta) \leq 1$

2. $\operatorname{Coh}(\alpha) \geq 0, \operatorname{Coh}(\beta) \geq 0$

3. $\operatorname{Coh}(\alpha) \leq 1, \operatorname{Coh}(\beta) \leq 1$

4. $\operatorname{Coh}(\alpha) \leq 1, \operatorname{Coh}(\beta) \geq 0$

Under these stimulus conditions, the number of recorded MEPs were certainly a hundred. Meanwhile, the number of MEP datapoints became smaller as the stimulus condition became stricter.

Figure 6 shows the distribution of the CV calculated from the MEP data. Each axis was determined in the same manner as shown in Figure 5. The color bar indicates the $\mathrm{CV}$ of MEP. We left these cells blank if the number of measured MEPs was less than ten in Figure 5. As shown in this figure, the MEP fluctuation in TMS was not suppressed under the weakest conditions at the center of the color map. Meanwhile, the MEP fluctuation was suppressed under stricter conditions than under the weakest conditions. Hence, this result indicates that there is a certain stimulus condition in which MEP fluctuation can be suppressed.
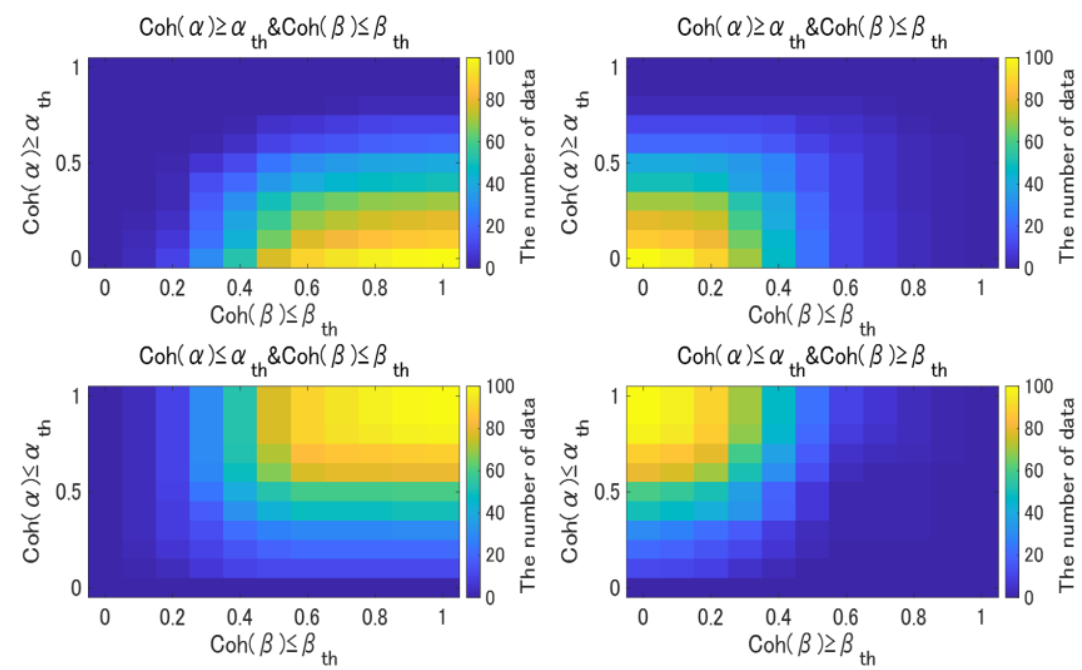

Fig. 5. Distribution of number of MEP data. 

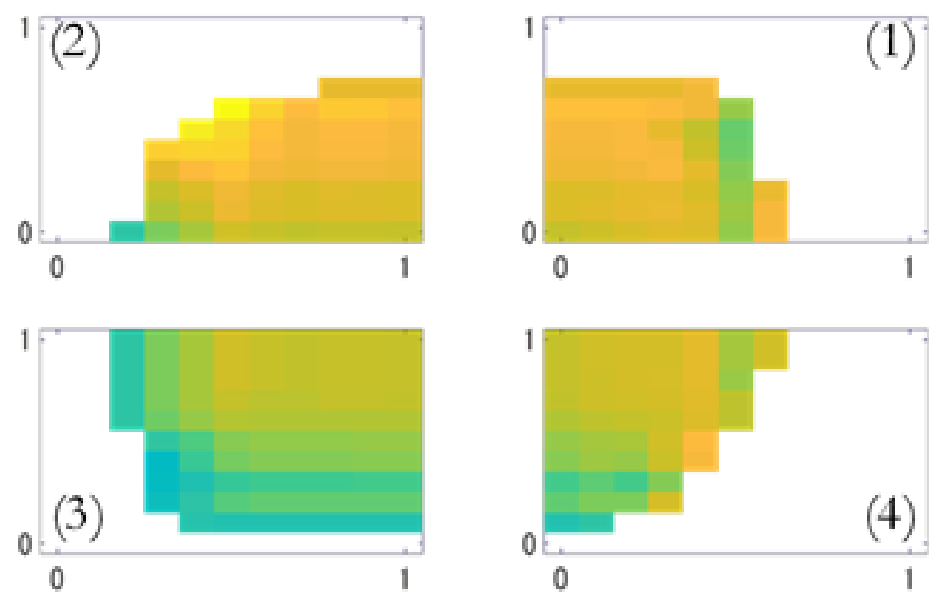

Fig. 6. Distribution of CV of MEP fluctuations for each stimulus condition.

\subsection{Color maps of $\mathrm{CV}$ of MEP}

Figure 7 shows the six color maps of the CV of the MEP measured from all subjects except for Subject 1. As shown in this figure, the CVs of MEP tended to decrease around the outside of the color map. We found that the coherence of EEG was related to the suppression of MEP fluctuations.

\subsection{Comparison of the $\mathrm{CV}$ of MEP between in the weakest and the strictest conditions}

As shown in Figure 7, we found that there is a relationship between the coherence value of EEG and the suppression amount of MEP fluctuation.

Here, we compared the results at the strictest and weakest conditions by determining each condition as conditional and non-conditional stimuli, respectively. We summarized the comparison of the CVs between conditional (the strictest) and nonconditional stimuli in all subjects in Table 1. The stimulus conditions are also listed in this table. Figure 8 shows a comparison of the CVs between the conditional and nonconditional stimuli. We found that the CVs of MEP fluctuation were lower for the conditional stimulus than for the unconditional stimulus.

The CVs of the MEPs were calculated at the stimulus conditions determined by the EEG coherence values. The variability of MEP amplitudes in each subject became small under some stimulus conditions obtained from the EEG coherence analysis. Although there is no certain condition for the lowest variability of MEP between subjects, the coherence value of cortical excitabilities measured from EEGs might be related to the changes in MEP variability. The CVs of MEP were significantly smaller in all subjects at the conditional stimulus, as shown in Figure 8. As listed in Table 1, 
all the conditions of the minimum CV of MEP, except for the condition of Subject 1, included a sign of equal to the threshold value or higher indicated with a red-colored symbol. This might indicate that a high similarity of EEG coherence is important in lowering fluctuations in MEP.
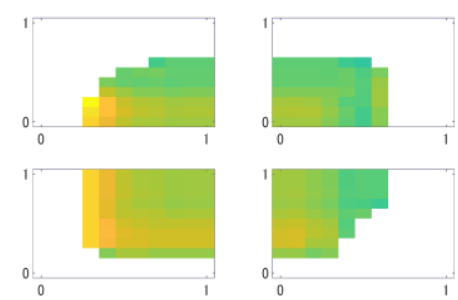

Subject 2
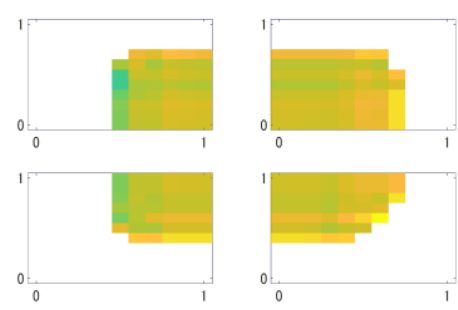

Subject 4
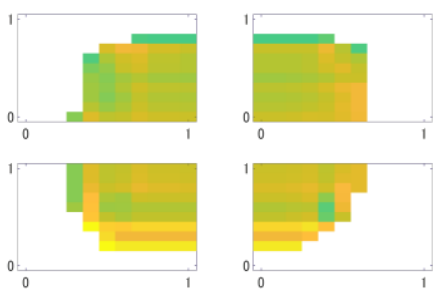

Subject 6

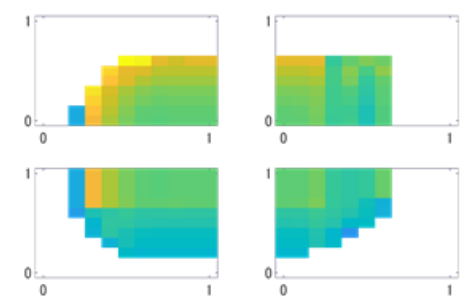

Subject 3
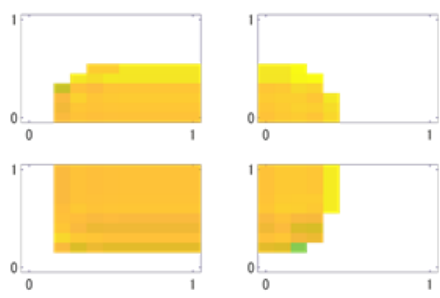

Subject 5
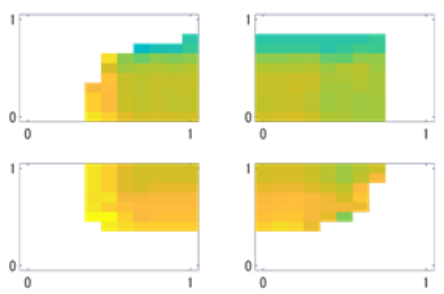

Subject 7

Fig. 7. Distributions of CV in Subject 2-7. 
Table 1. Summary of stimulus conditions

\begin{tabular}{|c|c|c|c|}
\hline Subject & Stimulus condition & Conditional & Non-conditional \\
\hline 1 & $\leqq 0.4 \& \leqq 0.3$ & 0.209 & 0.326 \\
\hline 2 & $\geqq 0.6 \& \geqq 0.5$ & 0.441 & 0.555 \\
\hline 3 & $\leqq 0.4 \& \geqq 0.4$ & 0.327 & 0.629 \\
\hline 4 & $\geqq 0.4 \& \leqq 0.5$ & 0.272 & 0.354 \\
\hline 5 & $\leqq 0.2 \& \geqq 0.2$ & 0.250 & 0.316 \\
\hline 6 & $\geqq 0.8 \& \geqq 0.0$ & 0.447 & 0.560 \\
\hline 7 & $\geqq 0.7 \& \leqq 0.7$ & 0.263 & 0.416 \\
\hline
\end{tabular}

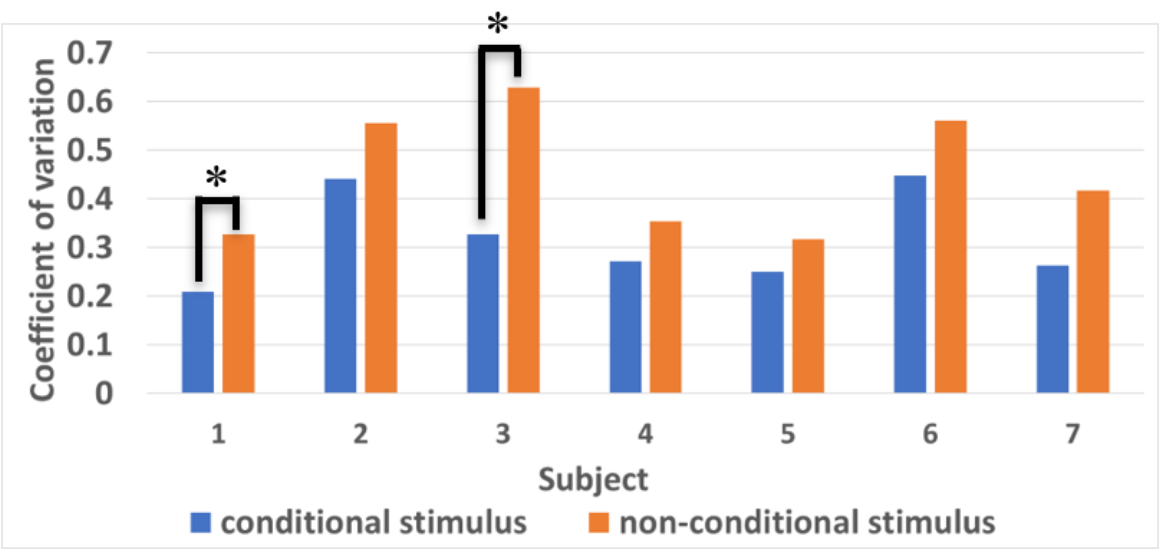

Fig. 8. Comparison of CVs of MEP between in conditional and non-conditional stimuli $(*: \mathrm{p}<0.05)$

\section{Conclusion}

In this study, we performed EEG coherence analysis and showed the distribution of the CV of MEP in TMS. The variability of MEP by a conditional stimulus was suppressed compared to that by an unconditional stimulus. This result indicated that the variability in MEP amplitude was related to cortical excitability. In another future study, we aim to develop a system for the suppression of MEP to observe stable MEP amplitudes for perioperative assessments. This system can enable precise evaluation of cortical excitability, and these results may improve the efficacy of patient prognostication.

\section{$5 \quad$ References}

[1] Barker, A.T., Jalinous, R., Freeston, I.L. Non-invasive magnetic stimulation of human motor cortex. Lancet, 1985, vol. 1, pp. 1106-1107. https://doi.org/10.1016/S0140-6736(85) 92413-4 
[2] Malcolm, M.P., Triggs, W.J., Light, K.E., Shechtman, O., Khandekar, G., Gonzalez, Rothi, L.J. Reliability of motor cortex transcranial magnetic stimulation in four muscle representations. Clinical Neurophysiology, 2006, vol. 117, pp. 1037-1046. https://doi.org/ 10.1016/j.clinph.2006.02.005

[3] Yamashita, A., Ishida, K., Matsumoto, M. Circumstances and Problems of Motor Evoked Potential Monitoring during Descending Thoracic and Thoracoabdominal Aortic Surgery. The journal of Japan Society for Clinical Anesthesia, 2014, vol. 34, pp. 868-874. https://doi.org/10.2199/jisca.34.868

[4] Takatani, T., Shigematsu, H., Motoyama, Y., Nakase, H., Kawaguchi, M. The usefulness of intraoperative check-lists and safety management for intraoperative neuro-monitoring. Japanese Journal of Clinical Neurophysiology, 2019, vol. 47, pp. 131-138 (in Japanese).

[5] Kiers, L., Cros, D, Chiappa, K.H., Fang, J. Variability of motor potentials evoked by transcranial magnetic stimulation. Electroencephalography and Clinical Neurophysiology/Evoked Potentials Section, 1993, vol. 89, pp. 415-423. https://doi.org/10.1016/01685597(93)90115-6

[6] Ellaway, P.H., Davey, N.J., Maskill, D.W., Rawlinson S.R., Lewis, H.S., Anissimova, N.P. Variability in the amplitude of skeletal muscle responses to magnetic stimulation of the motor cortex in man. Electroencephalography and Clinical Neurophysiology ,1998, vol. 109, pp. 104-113. https://doi.org/10.1016/S0924-980X(98)00007-1

[7] Wiethoff, S., Hamada, M., Rothwell, J.C. Variability in response to transcranial direct current stimulation of the motor cortex. Brain Stimulation, 2014, vol. 7, pp. 468-475. https://doi.org/10.1016/j.brs.2014.02.003

[8] Truccoloa, W.A., Ding, M., Knuth, K. H., Nakamura, R., Bresslera, L.S. Trial-to-trial variability of cortical evoked responses: implications for the analysis of functional connectivity. Clinical Neurophysiology, 2002, vol. 113, pp. 206-226. https://doi.org/10.1016/S13882457(01)00739-8

[9] Nakagawa, M., Sasaki, R., Tsuiki, S., Miyaguchi, S., Kojima, S., Saito, K., Inukai, Y., Onishi H. Effects of passive finger movement on cortical excitability. Frontiers Human Neuroscience, 2017, vol. 11, pp. 216. https://doi.org/10.3389/fnhum.2017.00216

[10] Barker, A.T., Freeston, I.L., Jalinous, R., Jarratt, J.A. Magnetic stimulation of the human brain and peripheral nervous system: an introduction and the results of an initial clinical evaluation. Neurosurgery, 1987, vol. 20, pp. 100-109. https://doi.org/10.1097/00006123$\underline{198701000-00024}$

[11] Rossini, P.M., Barker, A.T., Berardelli, A., Caramia, M.D., Caruso, G., Cracco, R.Q. et al. Non-invasive electrical and magnetic stimulation of the brain, spinal cord and roots: basic principles and procedures for routine clinical application. An updated report from an I.F.C.N. Committee. Clinical Neurophysiology, 1994, vol. 91, pp. 79-92. https://doi.org/10.1016/j.clinph.2015.02.001

[12] Liepert, J., Hallett, M., Samii, A., Oddo, D., Celnik, P., Cohen, L.G., Wassermann, E.M. Motor cortex excitability in patients with cerebellar degeneration. Clinical Neurophysiology, 2000, vol. 111, pp. 1157-1164. https://doi.org/10.1016/S1388-2457(00)00308-4

[13] Chipchase, L., Schabrun, S., Cohen, L., Hodges, P., Ridding, M., Rothwell, J., Taylor, J., Ziemann, Ulf. A checklist for assessing the methodological quality of studies using transcranial magnetic stimulation to study the motor system: an international consensus study. Clinical Neurophysiolosy, 2012, vol. 123, pp. 1698-1704. https://doi.org/10.1016/j.clin ph.2012.05.003

[14] Ogata, K., Nakazono, H., Uehara, T., Tobimatsu, S. Prestimulus cortical EEG oscillations can predict the excitability of the primary motor cortex. Brain Stimulation, 2019, vol. 12, pp. 1508-1516. https://doi.org/10.1016/j.brs.2019.06.013 
[15] Zarkowski, P., Shin, C.J., Dang, T., Russo, J., Avery, D. EEG and the variance of motor evoked potential amplitude. Clinical EEG and Neuroscience, 2006, vol. 37, pp. 247-251. https://doi.org/10.1177/155005940603700316

[16] Sauseng, P., Klimesch, W., Gerloff, C., Hummel, F.C. Spontaneous locally restricted EEG alpha activity determines cortical excitability in the motor cortex. Neuropsychologia, 2009, vol. 47, pp. 284-288. https://doi.org/10.1016/j.neuropsychologia.2008.07.021

[17] Mäki, H., Ilmoniemi, R.J. EEG oscillations and magnetically evoked motor potentials reflect motor system excitability in overlapping neuronal populations. Clinical Neurophysiology, 2010, vol. 121, pp. 492-501. https://doi.org/10.1016/j.clinph.2009.11.078

[18] Khademi, F., Royter, V, Gharabaghi, A. Distinct beta-band oscillatory circuits underlie corticospinal gain modulation. Cerebral Cortex, 2018, vol. 28, pp. 1502-1515. https://doi.org/10.1093/cercor/bhy016

[19] Katona, J., Kovari, A. EEG-based Computer Control Interface for Brain-Machine Interaction. International Journal of Online Engineering, 2015, vol. 11, pp. 43-48. http://doi.org/ $\underline{10.3991 / \text { ijoe.v11i6.5119 }}$

\section{Authors}

Keisuke Sasaki is a Ph.D. student of the Graduate School of Maebashi Institute of Technology and also is a clinical engineer working at Kanto Neurosurgical Hospital. $\mathrm{He}$ is an expert on MEP examination in perioperative assessments and developing a system for suppression for MEP fluctuation in TMS.

Yuki Fujishige is an undergraduate student of Maebashi Institute of Technology. His research interest is in the field of biomedical engineering and brain science.

Masato Odagaki received a master's degree from the Graduate School of Science and Engineering, Tokyo Denki University, Japan in 2004. He received a doctorate degree in engineering from the Graduate School of Advanced Science and Technology, Graduate School of Tokyo Denki University in 2007. He is currently an associate professor in the Department of System Life Engineering, Maebashi Institute of Technology. His research interests include the measurement and analysis of biological signals and bio-magnetism. He is a member of the Japanese Society for Medical and Biological Engineering, the Institute of Electrical Engineering of Japan, IEEE Engineering in Medicine and Biology Society, Japan Biomagnetism.

Article submitted 2021-03-10. Resubmitted 2021-04-18. Final acceptance 2021-04-19. Final version published as submitted by the authors. 